\title{
ON THE PROPAGATION OF TRANSIENTS THROUGH THERMOVISCOELASTIC MEDIA*
}

\author{
BY \\ M. F. McCARTHY (National University of Ireland, Galway, Ireland) \\ AND \\ T. B. MOODIE AND R. P. SAWATZKY (University of Alberta, Edmonton, Alberta, Canada)
}

\begin{abstract}
Summary. We examine the propagation of thermal and mechanical transients through general linear thermoviscoelastic media. A linear theory of heat conduction in deformable materials with memory is employed to study the one-dimensional problem of a homogeneous thermoviscoelastic half-space subjected to thermal and mechanical disturbances at its boundary. A ray series approach is used to generate asymptotic wave front expansions for the temperature, strain, and stress response of the medium to the disturbances. General properties of the propagation process are obtained simply and directly. As an example, we specialize the solution to the case of a medium where the conduction of heat obeys Cattaneo's law and the viscoelastic response is that of a standard linear solid. The propagation of transients through this material is depicted graphically for various values of the thermal and mechanical parameters.
\end{abstract}

1. Introduction. In the past few years, many researchers have sought to generalize the theory of heat conduction in rigid bodies to allow for the possibility of thermal disturbances propagating at a finite speed. A simple theory based on the ideas of Cattaneo [1] has been able to produce a finite propagation speed by using a modification of Fourier's law of heat conduction to derive a hyperbolic heat equation. Various attempts have been made to develop more general theories systematically by adopting the point of view of continuum thermodynamics. For example, Gurtin and Pipkin [2] employed this approach to formulate a general nonlinear theory of heat conduction in rigid materials with memory for which thermal disturbances propagate with finite velocities. A brief discussion of this theory, and others also based on the axioms of continuum thermodynamics, can be found in Agarwal [3].

Many of the theories of heat conduction for rigid materials can be extended in a natural way to deformable materials. For example, Lord and Shulman [4] incorporated Cattaneo's model of heat conduction into the classical dynamical theory of thermoelasticity to introduce within the theory the possibility of thermal disturbances propagating at a finite speed. Similarly, Chen and Gurtin [5] extended the theory of

${ }^{*}$ Received June 30, 1987.

(C) 1988 Brown University 
Gurtin and Pipkin to include deformable materials with memory. Typically, two speeds of propagation exist for longitudinal acceleration waves in deformable materials: a "first sound" speed predominantly mechanical in nature lying near the acoustical speed for the material and a "second sound" speed associated with a predominantly thermal wave.

The characteristics of thermoelastic wave propagation predicted by the various theories have been investigated in sorne detail, with much of the attention directed toward linear models. In particular, linear models involving the so-called MaxwellCattaneo relation in their description of heat conduction have been studied extensively; see [6]-[14]. The propagation characteristics of thermoviscoelastic materials have been discussed less frequently. Recently, McCarthy and O'Leary [15] investigated wave propagation in general linear thermoviscoelastic media by examining one-dimensional waves in thermoviscoelastic materials of the type considered by Gurtin [16]. The authors found the two possible speeds of propagation in such materials and derived the growth equation that governs the amplitude of discontinuities in the field variables at the wave fronts.

In this paper we extend the results of McCarthy and O'Leary to include the behavior of the field variables behind the wave fronts. We study the one-dimensional propagation of transients in homogeneous thermoviscoelastic half-spaces where the constitutive equations are those postulated by Gurtin [16]. Our approach toward the analysis of this problem is identical to the one adopted by Mainardi [17] and Moodie and Tait [18] for rigid heat conductors and by Sawatzky and Moodie [19] for thermoelastic ones. We develop asymptotic wave front expansions which not only yield the magnitude, decay, and propagation velocity of jumps in the field variables at the wave fronts but also provide a description of the behavior of the field variables behind the wave fronts.

2. Formulation. We consider a thermally conducting, isotropic, homogeneous, viscoelastic medium occupying the half-space $x>0$ with bounding surface at $x=0$. Initially the solid is undeformed, at rest, and at a uniform temperature. In subsequent departures from this reference state, the field variables are assumed to be functions of time and one space variable only. As well, the displacement gradient and relative temperature change are assumed to be very small compared with unity: that is,

$$
\left|\frac{\partial u}{\partial x}\right| \ll 1, \quad\left|T-T_{0}\right| \ll T_{0},
$$

where $u(x, t)$ is the displacement, $T(x, t)$ is the absolute temperature, and $T_{0}$ is the uniform reference temperature. Consequently, the field equations are linear. In the absence of body forces and external heat sources these equations are

$$
\begin{aligned}
& \rho \frac{\partial^{2} u}{\partial t^{2}}=\frac{\partial \sigma}{\partial x}, \\
& \frac{\partial e}{\partial t}=-\frac{\partial q}{\partial x},
\end{aligned}
$$


where $\sigma(x, t)$ is the stress, $q(x, t)$ the heat flux, $e(x, t)$ the internal energy per unit volume, and $\rho$ the constant mass density. The strain

$$
\varepsilon(x, t)=\frac{\partial u}{\partial x}
$$

will appear in the sequel as well.

The description of the continuum model is completed by the appropriate constitutive equations for $\sigma, e$, and $q$. We restrict our attention to media in which the stress and internal energy are linear functionals of the histories of the strain and temperature, and the heat flux is a linear functional of the history of the temperature gradient. Thus we choose our constitutive equations to be equivalent to those studied by McCarthy and O'Leary [15], namely

$$
\begin{aligned}
& \sigma(x, t)=\int_{-\infty}^{t} \beta_{1}(t-s) \frac{\partial \varepsilon(x, s)}{\partial s} d s-\int_{-\infty}^{t} \beta_{2}(t-s) \frac{\partial \theta(x, s)}{\partial s} d s, \\
& e(x, t)=\int_{-\infty}^{t} \beta_{4}(t-s) \frac{\partial \varepsilon(x, s)}{\partial s} d s+\int_{-\infty}^{t} \beta_{3}(t-s) \frac{\partial \theta(x, s)}{\partial s} d s, \\
& q(x, t)=-\int_{-\infty}^{t} \alpha(t-s) \frac{\partial \theta(x, s)}{\partial x} d s
\end{aligned}
$$

where

$$
\theta(x, t)=T(x, t)-T_{0}
$$

is the temperature difference. In Eqs. (2.5)-(2.7) $\beta_{1}$ is the stress-strain relaxation function, $\beta_{2}$ is the stress-temperature relaxation function, $\beta_{3}$ is the internal energytemperature relaxation function, $\beta_{4}$ is the internal energy-strain relaxation function, and $\alpha$ is the heat flux relaxation function. These relaxation functions are not all independent. Gurtin [16] has shown that

$$
\beta_{4}(t)=T_{0} \beta_{2}(t)
$$

Equations (2.5)-(2.7) describe a subclass of the materials examined by Gurtin [16], but our results may be extended straightforwardly to cover materials of the more general type.

The initial state we presume for the thermoviscoelastic medium leads to the following initial conditions:

$$
\theta(x, t)=\frac{\partial \theta(x, t)}{\partial t}=0, \quad u(x, t)=\frac{\partial u(x, t)}{\partial t}=0, \quad(x, t) \in(0, \infty) \times(-\infty, 0] .
$$

Transients are excited in the material as a consequence of thermal and mechanical disturbances generated at the boundary $x=0$. We suppose that these disturbances are produced by sudden changes in temperature and/or strain at $x=0$. Therefore the boundary conditions are taken to be

$$
\theta(0, t)=\theta_{1}(t), \quad \frac{\partial u(0, t)}{\partial v}=\varepsilon_{1}(t), \quad t \in(0, \infty)
$$


With the use of the initial conditions (2.10), Eq. (2.9), and a simple change of variable, the integrals in the constitutive Eqs. (2.5)-(2.7) may be integrated by parts to yield the set of equations

$$
\begin{gathered}
\sigma=\beta_{1}(0) \varepsilon-\beta_{2}(0) \theta+\int_{0}^{\infty} \frac{d \beta_{1}(s)}{d s} \varepsilon(x, t-s) d s-\int_{0}^{\infty} \frac{d \beta_{2}(s)}{d s} \theta(x, t-s) d s \\
e=T_{0} \beta_{2}(0) \varepsilon+\beta_{3}(0) \theta+T_{0} \int_{0}^{\infty} \frac{d \beta_{2}(s)}{d s} \varepsilon(x, t-s) d s+\int_{0}^{\infty} \frac{d \beta_{3}(s)}{d s} \theta(x, t-s) d s \\
q=-\int_{0}^{\infty} \alpha(s) \frac{\partial \theta(x, t-s)}{\partial x} d s
\end{gathered}
$$

When

$$
\beta_{1}(t)=\lambda+2 \mu, \quad \beta_{2}(t)=(3 \lambda+2 \mu) \alpha_{T}, \quad \beta_{3}(0)=\rho c_{v},
$$

where $\lambda$ and $\mu$ are the isothermal Lamé constants, $\alpha_{T}$ is the coefficient of linear thermal expansion, and $c_{v}$ is the instantaneous specific heat at constant volume, Eqs. (2.12)-(2.14) reduce to the constitutive equations for the thermoelastic medium considered by Sawatzky and Moodie [19]. As well, when

$$
\alpha(t)=\frac{\kappa}{\tau_{0}} e^{-t / \tau_{0}},
$$

where $\kappa$ is the coefficient of thermal conductivity and $\tau_{0}$ the thermal relaxation time, Eq. (2.14) becomes equivalent to the Maxwell-Cattaneo relation

$$
\tau_{0} \frac{\partial q}{\partial t}+q=-\kappa \frac{\partial \theta}{\partial x}
$$

In this paper we simply assume that the relaxation functions $\beta_{1}(t), \beta_{2}(t), \beta_{3}(t)$, and $\alpha(t)$ possess well-defined Taylor series expansions about $t=0$. Then

$$
\left.\begin{array}{ll}
\beta_{1}(t)=H(t) \sum_{i=0}^{\infty} \beta_{1, i}^{0} t^{i} / i !, & \beta_{2}(t)=H(t) \sum_{i=0}^{\infty} \beta_{2, i}^{0} t^{i} / i !, \\
\beta_{3}(t)=H(t) \sum_{i=0}^{\infty} \beta_{3, i}^{0} t^{i} / i !, & \alpha(t)=H(t) \sum_{i=0}^{\infty} \alpha_{i}^{0} t^{i} / i !,
\end{array}\right\}
$$

where

$$
\beta_{1, i}^{0}=\left.\frac{d^{i} \beta_{i}}{d t^{i}}\right|_{t=0}, \quad \beta_{2, i}^{0}=\left.\frac{d^{i} \beta_{2}}{d t^{i}}\right|_{t=0}, \quad \beta_{3, i}^{0}=\left.\frac{d^{i} \beta_{3}}{d t^{i}}\right|_{t=0}, \quad \alpha_{i}^{0}=\left.\frac{d^{i} \alpha}{d t^{i}}\right|_{t=0},
$$

and $H(t)$ is the Heaviside unit function. It is only necessary to specify the behavior of the relaxation functions near the origin since this completely determines the changes induced by the passage of the wave fronts.

Certain restrictions must be imposed on the relaxation functions as a consequence of physical considerations. We allow here only those thermoviscoelastic materials that admit to well-defined, finite discontinuity wave fronts propagating at finite speed. Therefore we require that

$$
\beta_{1,0}^{0}>0, \quad \beta_{2,0}^{0}>0, \quad \beta_{3,0}^{0}>0, \quad \alpha_{0}^{0}>0 .
$$


As well, we require that

$$
\beta_{1,1}^{0} \leq 0, \quad \beta_{2,1}^{0} \geq 0, \quad \beta_{3,1}^{0} \geq 0, \quad \alpha_{1}^{0} \leq 0 .
$$

The restrictions on $\beta_{1}$ are well known from the theory of viscoelasticity [20], while those on $\beta_{3}$ and $\alpha$ follow from the second law of thermodynamics [21]. It will be demonstrated in the following section that a restriction in addition to the one in (2.21) must be imposed on $\beta_{2,1}^{0}$ to ensure that discontinuities in temperature and strain at the wave fronts do not grow.

3. Ray series solution. It is convenient for the subsequent analysis to express the problem in nondimensional variables. To this end, we introduce the following dimensionless quantities:

$$
\left.\begin{array}{l}
\hat{x}=\left[\frac{\beta_{3,0}^{0}}{\kappa}\left(\beta_{1,0}^{0} / \rho\right)^{1 / 2}\right] x, \quad \hat{t}=\left[\frac{\beta_{3,0}^{0}}{\kappa}\left(\beta_{1,0}^{0} / \rho\right)\right] t, \\
\hat{u}=\left[\frac{\beta_{3,0}^{0}}{\kappa} \frac{\beta_{1,0}^{0}}{T_{0} \beta_{2,0}^{0}}\left(\beta_{1,0}^{0} / \rho\right)^{1 / 2}\right] u, \quad \hat{\theta}=\frac{\theta}{T_{0}}, \quad \hat{\sigma}=\frac{\sigma}{T_{0} \beta_{2,0}^{0}}, \\
\hat{e}=\frac{e}{T_{0} \beta_{3,0}^{0}}, \quad \hat{q}=\frac{q}{T_{0} \beta_{3,0}^{0}\left(\beta_{1,0}^{0} / \rho\right)^{1 / 2}}, \quad \hat{\varepsilon}=\left[\frac{\beta_{1,0}^{0}}{T_{0} \beta_{2,0}^{0}}\right] \varepsilon, \\
\hat{\beta}_{1}=\frac{\beta_{1}}{\beta_{1,0}^{0}}, \quad \hat{\beta}_{2}=\frac{\beta_{2}}{\beta_{2,0}^{0}}, \quad \hat{\beta}_{3}=\frac{\beta_{3}}{\beta_{3,0}^{0}}, \quad \hat{\alpha}=\frac{\alpha}{\beta_{3,0}^{0}\left(\beta_{1,0}^{0} / \rho\right)} .
\end{array}\right\}
$$

We also define the following dimensionless parameter, the thermoelastic coupling constant:

$$
\delta=\frac{\left(\beta_{2,0}^{0}\right)^{2} T_{0}}{\beta_{1,0}^{0} \beta_{3,0}^{0}} .
$$

In the sequel, unless otherwise indicated, dimensionless quantities will be used exclusively. Therefore, in the succeeding equations we drop the hats and retain the same letter to denote the corresponding dimensionless quantity.

After the appropriate nondimensional quantities from (3.1) are substituted into Eqs. (2.2)-(2.4) and (2.12)-(2.14), the resulting equations can be combined, with the use of (3.2), into the following pair of equations for $u$ and $\theta$ :

$$
\begin{gathered}
\frac{\partial^{2} u}{\partial t^{2}}-\frac{\partial^{2} u}{\partial x^{2}}=-\frac{\partial \theta}{\partial x}+\int_{0}^{\infty} \frac{d \beta_{1}(s)}{d s} \frac{\partial^{2} u(x, t-s)}{\partial x^{2}} d s-\int_{0}^{\infty} \frac{d \beta_{2}(s)}{d s} \frac{\partial \theta(x, t-s)}{\partial x} d s \\
\frac{\partial \theta}{\partial t}+\delta \frac{\partial^{2} u}{\partial x \partial t}+\delta \int_{0}^{\infty} \frac{d \beta_{2}(s)}{d s} \frac{\partial^{2} u(x, t-s)}{\partial x \partial t} d s+\int_{0}^{\infty} \frac{d \beta_{3}(s)}{d s} \frac{\partial \theta(x, t-s)}{\partial t} d s \\
=\int_{0}^{\infty} \alpha(s) \frac{\partial^{2} \theta(x, t-s)}{\partial x^{2}} d s
\end{gathered}
$$

The initial conditions $(2.10)$ and boundary conditions (2.11) retain the same appearance when they are expressed in dimensionless form:

$$
\begin{gathered}
\theta(x, t)=\frac{\partial \theta(x, t)}{\partial t}=0, \quad u(x, t)=\frac{\partial u(x, t)}{\partial t}=0, \quad(x, t) \in(0, \infty) \times(-\infty, 0], \\
\theta(0, t)=\theta_{1}(t), \quad \frac{\partial u(0, t)}{\partial x}=\varepsilon_{1}(t), \quad t \in(0, \infty) .
\end{gathered}
$$


The problem to be solved then consists of (3.3) and (3.4) augmented by conditions (3.5) and (3.6).

We apply the ray series method directly by representing the displacement field $u(x, t)$ and the temperature field $\theta(x, t)$ in terms of their asymptotic wave front expansions

$$
\begin{gathered}
u(x, t)=\sum_{j=1}^{\infty} U_{j}(x) F_{j}(t-S(x)), \quad U_{j} \equiv 0, j<1, \\
\theta(x, t)=\sum_{j=0}^{\infty} T_{j}(x) F_{j}(t-S(x)), \quad T_{j} \equiv 0, \quad j<0, \\
F_{j}^{\prime}=F_{j-1}, \quad j=1,2, \ldots,
\end{gathered}
$$

where the prime in (3.9) refers to differentiation with respect to the entire argument $t-S$. All of the $F_{j}$ for $j \geq 1$ can be determined from $F_{0}$ (the waveform) by successively integrating (3.9). For example, if $F_{0}(t)=H(t)$, then

$$
F_{j}(t)=t^{j} H(t) / j !
$$

Note that $F_{0}$ vanishes for negative argument in this case: that is, in front of the wave front whose equation is given by $t=S(x)$, where $S$ is the phase or eikonal function. For the $F_{j}$ given by (3.10), the coefficients $U_{j}$ and $T_{j}$ in (3.7) and (3.8) are the jump conditions for $u, \theta$ and their respective derivatives across the wave fronts.

The coefficients $U_{j}, T_{j}$ and the phase $S$ can be found by inserting the expansions (3.7) and (3.8) into Eqs. (3.3) and (3.4) and equating the coefficients of the functions $F_{j}$. Terms involving integrals are handled by replacing the dimensionless relaxation functions $\beta_{1}, \beta_{2}, \beta_{3}$, and $\alpha$ with their dimensionless Taylor series expansions and making use of the formula

$$
\int_{0}^{\infty}\left[\sum_{i=0}^{\infty} \gamma_{i} s^{i} / i !\right]\left[\sum_{j=0}^{\infty} V_{j} F_{j}(t-s)\right] d s=\sum_{j=1}^{\infty} F_{j}(t) \sum_{i=0}^{j-1} \gamma_{i} V_{j-1-i},
$$

where the coefficients $\gamma_{i}$ and $V_{j}$ are independent of $s$. The formula (3.11) is obtained via integration by parts and interchange of the order of summation. Thus, the following pair of equations is derived by inserting (3.7), (3.8), and the dimensionless version of (2.18) into (3.3) and (3.4), applying the result (3.11) to all the terms involving integrals, and equating the coefficients of $F_{j-1}$ :

$$
\begin{aligned}
& b_{11} U_{j+1}+b_{12} T_{j}+c_{11} U_{j}^{\prime}+c_{12} T_{j-1}^{\prime}=e_{11} U_{j}+e_{12} T_{j-1}+Q_{1, j-1}, \\
& b_{21} U_{j+1}+b_{22} T_{j}+c_{21} U_{j}^{\prime}+c_{22} T_{j-1}^{\prime}=e_{21} U_{j}+e_{22} T_{j-1}+Q_{2, j-1},
\end{aligned}
$$

for $j=0,1,2, \ldots$, where

$$
\begin{aligned}
b_{11}=1-\left(S^{\prime}\right)^{2}, \quad b_{12}=-S^{\prime}, \quad b_{21}=-\delta S^{\prime}, & b_{22}=1-\alpha_{0}^{0}\left(S^{\prime}\right)^{2}, \\
c_{11}=2 S^{\prime}, \quad c_{12}=1, \quad c_{21}=\delta, & c_{22}=2 \alpha_{0}^{0} S^{\prime}, \\
e_{11}=\beta_{1,1}^{0}\left(S^{\prime}\right)^{2}, \quad e_{12}=\beta_{2,1}^{0} S^{\prime}, \quad e_{21}=\delta \beta_{2,1}^{0} S^{\prime}, & e_{22}=\alpha_{1}^{0}\left(S^{\prime}\right)^{2}-\beta_{3,1}^{0},
\end{aligned}
$$


and

$$
\begin{aligned}
Q_{1, j} & =\sum_{i=0}^{j-1} L_{1, i} U_{j-i}+L_{2, i} T_{j-1-i}, \\
Q_{2, j} & =\sum_{i=0}^{j-1} \delta L_{2, i} U_{j-i}+L_{3, i} T_{j-1-i} .
\end{aligned}
$$

The linear differential operators $L_{1, i}, L_{2, i}$, and $L_{3, i}$ in (3.17) and (3.18) are defined by

$$
\left.\begin{array}{l}
L_{1, i} f \equiv \beta_{1, i}^{0} f^{\prime \prime}-2 \beta_{1, i+1}^{0} S^{\prime} f^{\prime}+\beta_{1, i+2}^{0}\left(S^{\prime}\right)^{2} f, \\
L_{2, i} f \equiv-\left(\beta_{2, i+1}^{0} f^{\prime}-\beta_{2, i+2}^{0} S^{\prime} f\right), \\
L_{3, i} f \equiv \alpha_{i}^{0} f^{\prime \prime}-2 \alpha_{i+1}^{0} S^{\prime} f^{\prime}+\left[\alpha_{i+2}^{0}\left(S^{\prime}\right)^{2}-\beta_{3, i+2}^{0}\right] f .
\end{array}\right\}
$$

The terms involving $S^{\prime \prime}$ have been omitted from (3.19) since it is shown subsequently that $S^{\prime}$ is constant.

The first of the equations in (3.12) and (3.13), those for $j=0$, are independent of the terms that were omitted:

$$
\left.\begin{array}{l}
b_{11} U_{1}+b_{12} T_{0}=0 \\
b_{21} U_{1}+b_{22} T_{0}=0
\end{array}\right\}
$$

In order that these equations have a nontrivial solution for $U_{1}$ and $T_{0}$, it is necessary that

$$
b_{11} b_{22}-b_{12} b_{21}=0
$$

Equation (3.21) is the eikonal equation for the thermoviscoelastic material. With the use of (3.14), the eikonal equation can be written as

$$
\left[1-\left(S^{\prime}\right)^{2}\right]\left[1-\alpha_{0}^{0}\left(S^{\prime}\right)^{2}\right]-\delta\left(S^{\prime}\right)^{2}=0
$$

Equation (3.22) is identical to the eikonal equation for a linear thermoelastic material [19].

As disturbances leave the boundary at $x=0$ and travel into the half-space $x>0$, they generate two wave fronts located at

$$
t=S_{1}(x)=S_{1}^{\prime} x \quad \text { and } \quad t=S_{2}(x)=S_{2}^{\prime} x
$$

where

$$
\left.\begin{array}{c}
S_{1}^{\prime}=\left[\frac{1}{2 \alpha_{0}^{0}}\left(1+\delta+\alpha_{0}^{0}+\Gamma^{1 / 2}\right)\right]^{1 / 2}, \\
S_{2}^{\prime}=\left[\frac{1}{2 \alpha_{0}^{0}}\left(1+\delta+\alpha_{0}^{0}-\Gamma^{1 / 2}\right)\right]^{1 / 2}, \\
\Gamma=\left(\delta+\alpha_{0}^{0}-1\right)^{2}+4 \delta .
\end{array}\right\}
$$

The wave fronts travel through the medium with constant speeds $v_{1}=1 / S_{1}^{\prime}$ and $v_{2}=1 / S_{2}^{\prime}$ respectively. As $S_{1}^{\prime}>S_{2}^{\prime}$, the faster of the two waves travels with velocity $v_{2}$ while the slower travels with velocity $v_{1}$. Moreover, it follows from (3.22) and (3.24) that $\left(S_{1}^{\prime}\right)^{2}>1$ and $\left(S_{2}^{\prime}\right)^{2}<1$. Therefore the velocity of the faster (slower) wave 
is greater (less) than the unit dimensionless velocity, the instantaneous isothermal velocity of longitudinal elastic waves.

It is apparent from the preceding analysis that the asymptotic wave front expansions for $u$ and $\theta$ consist of the sum of expansions about each wave front. In order to find the coefficients $U_{j}, T_{j}$ for each expansion, we return to the so-called transport equations (3.12) and (3.13). When $j=0$, it follows from (3.20) and (3.21) that

$$
U_{1}(x)=-\frac{b_{12}}{b_{11}} T_{0}(x)=\frac{S^{\prime}}{1-\left(S^{\prime}\right)^{2}} T_{0}(x) .
$$

When $j>0,(3.12)$ and (3.13) do not form a linearly independent set of equations for $U_{j+1}, T_{j}$ either. Both $U_{j+1}$ and $T_{j}$ can be eliminated from (3.12) and (3.13) with the use of (3.21). The resulting equation,

$$
a_{11} U_{j}^{\prime}+a_{12} T_{j-1}^{\prime}=a_{21} U_{j}+a_{22} T_{j-1}+b_{11} Q_{2, j-1}-b_{21} Q_{1, j-1},
$$

for $j=1,2, \ldots$, where

$$
\left.\begin{array}{l}
a_{11}=b_{11} c_{21}-b_{21} c_{11}=\delta\left(1+\left(S^{\prime}\right)^{2}\right), \\
a_{12}=b_{11} c_{22}-b_{21} c_{12}=S^{\prime}\left[\delta+2 \alpha_{0}^{0}\left(1-\left(S^{\prime}\right)^{2}\right)\right], \\
a_{21}=b_{11} e_{21}-b_{21} e_{11}=\delta S^{\prime}\left[\beta_{1,1}^{0}\left(S^{\prime}\right)^{2}+\beta_{2,1}^{0}\left(1-\left(S^{\prime}\right)^{2}\right)\right], \\
a_{22}=b_{11} e_{22}-b_{21} e_{12}=\delta \beta_{2,1}^{0}\left(S^{\prime}\right)^{2}-\left(1-\left(S^{\prime}\right)^{2}\right)\left(\beta_{3,1}^{0}-\alpha_{1}^{0}\left(S^{\prime}\right)^{2}\right),
\end{array}\right\}
$$

can be viewed as a compatibility condition for the pair of equations (3.12) and (3.13).

By putting $j=1$ in (3.27) and using (3.26), the following first-order linear differential equation is obtained for $T_{0}$ :

$$
T_{0}^{\prime}+w T_{0}=0
$$

where

$$
w=-\frac{\left(b_{11} a_{22}-b_{12} a_{21}\right)}{\left(b_{11} a_{12}-b_{12} a_{11}\right)}
$$

The solution is

$$
T_{0}(x)=\bar{T}_{0} e^{-w x},
$$

where $\bar{T}_{0}=T_{0}(0)$. A second basic fact regarding the propagation characteristics of a thermoviscoelastic medium emerges from Eqs. (3.31) and (3.26): a discontinuous change in temperature or strain decays at each wave front provided the rate constant $w$ in (3.30) is positive. McCarthy and O'Leary [15] arrived at the same conclusion.

The dependence of the sign of $w$ on the relaxation functions $\beta_{1}, \beta_{2}, \beta_{3}$, and $\alpha$ can be ascertained by inserting (3.14) and (3.28) into (3.30). After some calculations, the following expression for $w$ can be obtained:

$$
\begin{aligned}
w= \pm \frac{\left(\left(S^{\prime}\right)-1\right)}{2 S^{\prime} \Gamma^{1 / 2}}\left[\alpha_{1}^{0}\left(S^{\prime}\right)^{2}\right. & -\beta_{3,1}^{0}+\frac{1}{\delta}\left(1-\alpha_{0}^{0}\left(S^{\prime}\right)^{2}\right)^{2} \beta_{1,1}^{0} \\
& \left.+2\left(1-\alpha_{0}^{0}\left(S^{\prime}\right)^{2}\right) \beta_{2,1}^{0}\right] .
\end{aligned}
$$

the $+/-$ signs in (3.32) are associated with the faster/slower wave fronts, respectively. Therefore $w>0$ whenever

$$
\alpha_{1}^{0}\left(S^{\prime}\right)^{2}-\beta_{3,1}^{0}+\frac{1}{\delta}\left(1-\alpha_{0}^{0}\left(S^{\prime}\right)^{2}\right)^{2} \beta_{1,1}^{0}+2\left(1-\alpha_{0}^{0}\left(S^{\prime}\right)^{2}\right) \beta_{2,1}^{0}<0 .
$$


In light of (2.21), the only term in (3.33) that possibly can be positive is $2(1-$ $\left.\alpha_{0}^{0}\left(S^{\prime}\right)^{2}\right) \beta_{2,1}^{0}$ : this term is nonnegative at the faster wave front. Thus, $\beta_{2,1}^{0}$ must be bounded from above for (3.33) to hold at both wave fronts. For example, the following upper bound on $\beta_{2,1}^{0}$, namely

$$
\beta_{2,1}^{0} \leq-\frac{\left(1-\left(S_{2}^{\prime}\right)^{2}\right)}{2 \delta} \alpha_{1}^{0}
$$

guarantees that (3.33) is never violated, as long as $\beta_{1,1}^{0}, \beta_{3,1}^{0}$, and $\alpha_{1}^{0}$ do not vanish simultaneously.

The next set of coefficients, $U_{2}$ and $T_{1}$, can be obtained from (3.12), (3.13), and (3.27) by the same procedure that yielded $U_{1}$ and $T_{0}$. We find $U_{2}$ in terms of $T_{1}$ from (3.12) and (3.13) when $j=1$ and use this result in (3.27) when $j=2$ to derive a first-order linear differential equation for $T_{1}$. The process can be repeated indefinitely to construct as many coefficients $U_{j+1}, T_{j}$ as desired. However, as the algebraic manipulations involved become increasingly tedious with each increment of $j$, we adopt an alternative scheme.

We seek solutions for $U_{j}(x), T_{j}(x)$ that are of the form

$$
\begin{array}{ll}
U_{j}(x)=e^{-w x} \sum_{k=0}^{j-1} u_{k j} x^{k} / k !, & u_{k j}=0, k>j-1, \\
T_{j}(x)=e^{-w x} \sum_{k=0}^{j} t_{k j} x^{k} / k !, & t_{k j}=0, k>j .
\end{array}
$$

When the trial solutions (3.34) and (3.35) are inserted into (3.12), (3.13), and (3.27) and the coefficients of $x^{k}$ equated, the following difference equations for $u_{k, j+1}$ and $t_{k j}$ are produced:

$$
\begin{gathered}
b_{11} u_{k, j+1}+b_{12} t_{k j}=-\left(c_{11} E_{w}-e_{11}\right) u_{k j}-\left(c_{12} E_{w}-e_{12}\right) t_{k, j-1}+q_{1, k, j-1}, \\
b_{21} u_{k, j+1}+b_{22} t_{k j}=-\left(c_{21} E_{w}-e_{21}\right) u_{k j}-\left(c_{22} E_{w}-e_{22}\right) t_{k, j-1}+q_{2, k, j-1}, \\
\left(a_{11} E_{w}-a_{21}\right) u_{k, j+1}+\left(a_{12} E_{w}-a_{22}\right) t_{k j}=b_{11} q_{2, k, j}-b_{21} q_{1, k, j},
\end{gathered}
$$

for $k=0,1,2, \ldots, j, j=0,1,2, \ldots$ In the derivation of $(3.38), j$ has been replaced with $j+1$. The weighted difference operator $E_{w}$ is defined by

$$
E_{w} f_{k j}=f_{k+1, j}-w f_{k j}
$$

and

$$
\begin{aligned}
q_{1, k, j} & =\sum_{i=0}^{j-k-1} M_{1, i} u_{k, j-i}+M_{2, i} t_{k, j-1-i}, \\
q_{2, k, j} & =\sum_{i=0}^{j-k-1} \delta M_{2, i} u_{k, j-i}+M_{3, i} t_{k, j-1-i} .
\end{aligned}
$$

The linear difference operators $M_{1, i}, M_{2, i}$, and $M_{3, i}$ in (3.40) and (3.41) are defined by

$$
\left.\begin{array}{l}
M_{1, i} \equiv \beta_{1, i}^{0} E_{w^{\prime}}^{2}-2 \beta_{1, i+1}^{0} S^{\prime} E_{w}+\beta_{1, i+2}^{0}\left(S^{\prime}\right)^{2}, \\
M_{2, i} \equiv-\left(\beta_{2, i+1}^{0} E_{w}-\beta_{2, i+2}^{0} S^{\prime}\right), \\
M_{3, i} \equiv \alpha_{i}^{0} E_{w}^{2}-2 \alpha_{i+1}^{0} S^{\prime} E_{w}+\left(\alpha_{i+2}^{0}\left(S^{\prime}\right)^{2}-\beta_{3, i+2}^{0}\right),
\end{array}\right\}
$$


as they are related to the linear differential operators $L_{1, i}, L_{2, i}$, and $L_{3, i}$ given in (3.19).

Equations (3.36) and (3.37) are linearly dependent. Therefore only one of them, say (3.36), is required in conjunction with (3.38) to obtain the two unknowns $u_{k, j+1}$ and $t_{k j}$. The elimination of $u_{k, j+1}$ from (3.36) and (3.38) yields a formula for $t_{k j}$ that holds for $k=1,2, \ldots, j, j=1,2, \ldots$ :

$$
\begin{array}{r}
t_{k j}=\left\{\left(a_{11} E_{w}-a_{21}\right)\left[\left(c_{11} E_{w}-e_{11}\right) u_{k-1, j}+\left(c_{12} E_{w}-e_{12}\right) t_{k-1, j-1}-q_{1, k-1, j-1}\right]\right. \\
\left.+b_{11}\left(b_{11} q_{2, k-1, j}-b_{21} q_{1, k-1, j}\right)\right\} /\left(b_{11} a_{12}-b_{12} a_{11}\right) .
\end{array}
$$

The formula (3.43) for $t_{k j}$, in turn, can be inserted into (3.36) to produce a formula for $u_{k j}$ that is valid for $k=1,2, \ldots, j-1, j=2,3, \ldots$ :

$$
u_{k j}=-\frac{1}{b_{11}}\left[b_{12} t_{k, j-1}+\left(c_{11} E_{w}-e_{11}\right) u_{k, j-1}+\left(c_{12} E_{w}-e_{12}\right) t_{k, j-2}-q_{1, k, j-2}\right] .
$$

The formulas (3.43) and (3.44) do not generate $t_{0 j}=T_{j}(0)=\bar{T}_{j}$ or $u_{0 j}=U_{j}(0)=$ $\bar{U}_{j}$. Equations (3.36) and (3.38) are not capable of determining $\bar{U}_{j}$ and $\bar{T}_{j}$ completely. A single relationship between $\bar{U}_{j+1}$ and $\bar{T}_{j}$ arises, however, from (3.36) when $k=0$ :

$$
\begin{aligned}
b_{11} \bar{U}_{j+1}+b_{12} \bar{T}_{j}+c_{11} u_{1, j}-\left(w c_{11}+e_{11}\right) \bar{U}_{j} & +c_{12} t_{1, j-1} \\
& -\left(w c_{12}+e_{12}\right) \bar{T}_{j-1}-q_{1,0, j-1}=0,
\end{aligned}
$$

for $j=0,1,2, \ldots$. Equation (3.45) must be supplemented by the given initial and boundary conditions in order to finish the calculation of $\bar{U}_{j+1}$ and $\bar{T}_{j}$.

In summary, then, the coefficients $t_{k j}$ of (3.35) are given recursively by

$$
t_{k j}=\left\{\begin{array}{l}
{\left[\left(a_{11} E_{w}-a_{21}\right)\left[\left(c_{11} E_{w}-e_{11}\right) u_{k-1, j}+\left(c_{12} E_{w}-e_{12}\right) t_{k-1, j-1}-q_{1, k-1, j-1}\right]\right.} \\
\left.\quad+b_{11}\left(b_{11} q_{2, k-1, j}-b_{21} q_{1, k-1, j}\right)\right] /\left(b_{11} a_{12}-b_{12} a_{11}\right), \quad 1 \leq k \leq j, \\
\bar{T}_{j}, \quad k=0, \quad j \geq 0, \\
0, \quad k<0 \text { or } k>j,
\end{array}\right.
$$

and the coefficients $u_{k j}$ of (3.34) are given recursively by

$$
u_{k j}= \begin{cases}-\frac{1}{b_{11}}\left[b_{12} t_{k, j-1}+\left(c_{11} E_{w}-e_{11}\right) u_{k, j-1}+\left(c_{12} E_{w}-e_{12}\right) t_{k, j-2}-q_{1, k, j-2}\right), & 1 \leq k \leq j-1, \\ \bar{U}_{j}, & k=0, \quad j \geq 1, \\ 0, & k<0 \text { or } k>j-1 .\end{cases}
$$

In order to complete the solution of the problem posed in (3.3)-(3.6), we need to introduce the source conditions (3.6). Consider the following two simple examples of source conditions, namely

$$
\theta(0, t)=H(t), \quad \frac{\partial u(0, t)}{\partial x}=0
$$

and

$$
\theta(0, t)=0, \quad \frac{\partial u(0, t)}{\partial x}=H(t)
$$


We denote the problem associated with each of these conditions by problem 1 and problem 2, respectively. The solution of the problem with general source conditions can be obtained from the solutions of these two canonical problems through appropriate superposition and the use of Duhamel's theorem.

Let $u^{1}, \theta^{1}$ and $u^{2}, \theta^{2}$ be the wave front expansions for the displacement field and the temperature field near $t=S_{1}^{\prime} x$ and $t=S_{2}^{\prime} x$, respectively. Substitution of the sums of the expansions at each wave front, as determined by (3.7) and (3.8), into (3.48) and (3.49) and subsequent use of (3.34) and (3.35) results in the set of equations

$$
\begin{gathered}
\sum_{j=0}^{\infty} \sum_{l=1}^{2} \bar{T}_{j}^{l} F_{j}(t)= \begin{cases}H(t) & \text { for problem 1, } \\
0, & \text { for problem 2, }\end{cases} \\
\sum_{j=0}^{\infty} \sum_{l=1}^{2}\left(u_{1, j}^{l}-w_{l} \bar{U}_{j}^{l}-S_{l}^{\prime} \bar{U}_{j+1}^{l}\right) F_{j}(t)= \begin{cases}0, & \text { for problem 1, } \\
H(t), & \text { for problem 2, }\end{cases}
\end{gathered}
$$

where the index $l$ indicates that the corresponding quantity is associated with the wave front at $t=S_{l}^{\prime} x$. Therefore we choose

$$
F_{0}(t)=H(t)
$$

and

$$
\left.\begin{array}{c}
\bar{T}_{j}^{1}+\bar{T}_{j}^{2}=0, \\
\left(u_{1, j}^{1}-w_{1} \bar{U}_{j}^{1}-S_{1}^{\prime} \bar{U}_{j+1}^{1}\right)+\left(u_{1, j}^{2}-w_{2} \bar{U}_{j}^{2}-S_{2}^{\prime} \bar{U}_{j+1}^{2}\right)=0,
\end{array}\right\}
$$

$j=1,2, \ldots$, for each problem, together with

$$
\begin{array}{ll}
\bar{T}_{0}^{1}+\bar{T}_{0}^{2}=1, & S_{1}^{\prime} \bar{U}_{1}^{1}+S_{2}^{\prime} \bar{U}_{1}^{2}=0, \quad \text { for problem } 1 \\
\bar{T}_{0}^{1}+\bar{T}_{0}^{2}=0, & S_{1}^{\prime} \bar{U}_{1}^{1}+S_{2}^{\prime} \bar{U}_{1}^{2}=-1, \quad \text { for problem } 2 .
\end{array}
$$

With $F_{0}(t)$ given by (3.52), the remaining $F_{j}(t)$ are given by (3.10). We observe that if $F_{0}(t)=f(t)$, where $f(t)$ is arbitrary, then the remaining $F_{j}(t)$ may be found from those for $f(t)=H(t)$ by Duhamel's theorem [22, p. 512]; that is,

$$
F_{j}(t)=\frac{H(t)}{j !} \frac{\partial}{\partial t} \int_{0}^{t}(t-s)^{j} f(s) d s
$$

The solution of (3.53) subject to (3.45) generates recursion relations for the coefficients $\bar{T}_{j}, \bar{U}_{j+1}$ for $j=1,2, \ldots$ After some simplification, these relations can be 
expressed as

$$
\begin{aligned}
\bar{T}_{1}^{1}= & -\bar{T}_{j}^{2} \\
= & -\alpha_{0}^{0} \Gamma^{-1 / 2}\left\{S _ { 1 } ^ { \prime } ( 1 - ( S _ { 2 } ^ { \prime } ) ^ { 2 } ) \left[-c_{11}^{1} u_{1, j}^{1}+\left(w_{1} c_{11}^{1}+e_{11}^{1}\right) \bar{U}_{j}^{1}\right.\right. \\
& \left.-c_{12}^{1} t_{1, j-1}^{1}+\left(w_{1} c_{12}^{1}+e_{12}^{1}\right) \bar{T}_{j-1}^{1}+q_{1,0, j-1}^{1}\right] \\
& -S_{2}^{\prime}\left(\left(S_{1}^{\prime}\right)^{2}-1\right)\left[-c_{11}^{2} u_{1, j}^{2}+\left(w_{2} c_{11}^{2}+e_{11}^{2}\right) \bar{U}_{j}^{2}-c_{12}^{2} t_{1, j-1}^{2}\right. \\
& \left.+\left(w_{2} c_{12}^{2}+e_{12}^{2}\right) \bar{T}_{j-1}^{2}+q_{1,0, j-1}^{2}\right] \\
& \left.+\left(\left(S_{1}^{\prime}\right)^{2}-1\right)\left(1-\left(S_{2}^{\prime}\right)^{2}\right)\left[u_{1, j}^{1}-w_{1} \bar{U}_{j}^{1}+u_{1, j}^{2}-w_{2} \bar{U}_{j}^{2}\right]\right\}, \\
\bar{U}_{j+1}^{1}= & -\left[S_{1}^{\prime} \bar{T}_{j}^{1}-c_{11}^{1} u_{1, j}^{1}+\left(w_{1} c_{11}^{1}+e_{11}^{1}\right) \bar{U}_{j}^{1}-c_{12}^{1} t_{1, j-1}^{1}\right. \\
& \left.+\left(w_{1} c_{12}^{1}+e_{12}^{1}\right) \bar{T}_{j-1}^{1}+q_{1,0, j-1}^{1}\right] /\left(\left(S_{1}^{\prime}\right)^{2}-1\right), \\
\bar{U}_{j+1}^{2}= & {\left[S_{2}^{\prime} \bar{T}_{j}^{2}-c_{11}^{2} u_{1, j}^{2}+\left(w_{2} c_{11}^{2}+e_{11}^{2}\right) \bar{U}_{j}^{2}-c_{12}^{2} t_{1, j-1}^{2}\right.} \\
& \left.+\left(w_{2} c_{12}^{2}+e_{12}^{2}\right) \bar{T}_{j-1}^{2}+q_{1,0, j-1}^{2}\right] /\left(1-\left(S_{2}^{\prime}\right)^{2}\right) .
\end{aligned}
$$

These recursion relations are, of course, the same for each problem. The initial coefficients $\bar{T}_{0}^{1}, \bar{T}_{0}^{2}, \bar{U}_{1}^{1}$, and $\bar{U}_{1}^{2}$ are obtained for each problem from the solutions of (3.54) and (3.55), respectively, subject to (3.45). Their simplified forms are

$$
\left.\begin{array}{l}
\bar{T}_{0}^{1}=\alpha_{0}^{0} \Gamma^{-1 / 2}\left(S_{2}^{\prime}\right)^{2}\left(\left(S_{1}^{\prime}\right)^{2}-1\right), \quad \bar{T}_{0}^{2}=\alpha_{0}^{0} \Gamma^{-1 / 2}\left(S_{1}^{\prime}\right)^{2}\left(1-\left(S_{2}^{\prime}\right)^{2}\right), \\
\bar{U}_{1}^{1}=-\alpha_{0}^{0} \Gamma^{-1 / 2} S_{1}^{\prime}\left(S_{2}^{\prime}\right)^{2}, \quad \bar{U}_{1}^{2}=\alpha_{0}^{0} \Gamma^{-1 / 2}\left(S_{1}^{\prime}\right)^{2} S_{2}^{\prime}, \quad \text { for problem 1, }
\end{array}\right\}
$$

and

$$
\left.\begin{array}{l}
\bar{T}_{0}^{1}=-\bar{T}_{0}^{2}=\alpha_{0}^{0} \Gamma^{-1 / 2}\left(\left(S_{1}^{\prime}\right)^{2}-1\right)\left(1-\left(S_{2}^{\prime}\right)^{2}\right), \\
\bar{U}_{1}^{1}=-\alpha_{0}^{0} \Gamma^{-1 / 2} S_{1}^{\prime}\left(1-\left(S_{2}^{\prime}\right)^{2}\right), \\
\bar{U}_{1}^{2}=-\alpha_{0}^{0} \Gamma^{-1 / 2} S_{2}^{\prime}\left(\left(S_{1}^{\prime}\right)^{2}-1\right), \text { for problem } 2 .
\end{array}\right\}
$$

By combining the above results, we find that the solutions of the two canonical problems associated with the source conditions (3.48) and (3.49) can be written in each case as

$$
\begin{aligned}
& u(x, t)=\sum_{l=1}^{2} e^{-w_{l} x}\left[\sum_{j=1}^{\infty} \frac{\left(t-S_{l}^{\prime} x\right)^{j}}{j !} \sum_{k=0}^{j-1} u_{k j}^{l} x^{k} / k !\right] H\left(t-S_{l}^{\prime} x\right), \\
& \theta(x, t)=\sum_{l=1}^{2} e^{-w_{l} x}\left[\sum_{j=0}^{\infty} \frac{\left(t-S_{l}^{\prime} x\right)^{j}}{j !} \sum_{k=0}^{j} t_{k j}^{l} x^{k} / k !\right] H\left(t-S_{l}^{\prime} x\right),
\end{aligned}
$$

where the $S_{l}^{\prime}$ are given by (3.24) and the corresponding $w_{l}$ by (3.30). The coefficients $t_{k j}^{l}$ and $u_{k j}^{l}$ are calculated recursively from (3.46) and (3.47), with $\bar{T}_{j}^{l}$ and $\bar{U}_{j}^{l}$ provided by Eq. (3.57). For problem 1 the starting values $\bar{T}_{0}^{l}$ and $\bar{U}_{1}^{l}$ are presented in (3.58), whereas for problem 2 these quantities are found in (3.59). The strain corresponding 
to the displacement $(3.60)$ is

$$
\varepsilon(x, t)=\sum_{l=1}^{2} e^{-w_{l} x}\left[\sum_{j=0}^{\infty} \frac{\left(t-S_{l}^{\prime} x\right)^{j}}{j !} \sum_{k=0}^{j} \frac{\left(u_{k+1, j}^{l}-w_{l} u_{k j}^{l}-S_{l}^{\prime} u_{k, j+1}^{l}\right) x^{k}}{k !}\right] H\left(t-S_{l}^{\prime} x\right) .
$$

As noted earlier, the solutions given in (3.60) and (3.61) can be used to construct the solutions to problems involving more general source conditions as a consequence of Duhamel's theorem, that is, via (3.56).

The discontinuities in temperature, strain, and stress at the boundary $x=0$ introduced by (3.48) and (3.49) evolve into discontinuities in these variables across the two wave fronts $t=S_{1}^{\prime} x$ and $t=S_{2}^{\prime} x$. The amplitudes of the discontinuities in temperature and strain are, from (3.61) and (3.62),

$$
\begin{aligned}
& {[\theta]_{t=S_{l}^{\prime} x}=\bar{T}_{0}^{l} e^{-w_{l} x},} \\
& {[\varepsilon]_{t=S_{l}^{\prime} x}=-S_{l}^{\prime} \bar{U}_{1}^{l} e^{-w_{l} x} .}
\end{aligned}
$$

The amplitude of the discontinuity in stress follows from the dimensionless version of the constitutive equation (2.12). Thus

$$
[\sigma]_{t=S_{l}^{\prime} x}=[\varepsilon]_{t=S_{l}^{\prime} x}-[\theta]_{t=S_{l}^{\prime} x} .
$$

In Eqs. (3.63)-(3.65), the discontinuity in a function $f(x, t)$ across a wave front at $t=S(x)$ is defined to be

$$
\left.[f]_{t=S(x)} \equiv f(x, t)\right|_{t=S(x)^{+}}-\left.f(x, t)\right|_{t=S(x)^{-}} .
$$

The jumps in temperature, strain, and stress across the wave fronts can be expressed in greater detail for each problem with the use of (3.58) and (3.59). The results for temperature and strain are obvious. In the case of stress they are

$$
\left.\begin{array}{l}
{[\sigma]_{t=S_{1}^{\prime} x}=\alpha_{0}^{0} \Gamma^{-1 / 2}\left(S_{2}^{\prime}\right)^{2} e^{-w_{1} x},} \\
{[\sigma]_{t=S_{2}^{\prime} x}=-\alpha_{0}^{0} \Gamma^{-1 / 2}\left(S_{1}^{\prime}\right)^{2} e^{-w_{2} x}, \text { for problem } 1,}
\end{array}\right\}
$$

and

$$
\left.\begin{array}{l}
{[\sigma]_{t=S_{1}^{\prime} x}=\alpha_{0}^{0} \Gamma^{-1 / 2}\left(1-\left(S_{2}^{\prime}\right)^{2}\right) e^{-w_{1} x},} \\
{[\sigma]_{t=S_{2}^{\prime} x}=\alpha_{0}^{0} \Gamma^{-1 / 2}\left(\left(S_{1}^{\prime}\right)^{2}-1\right) e^{-w_{2} x}, \text { for problem 2. }}
\end{array}\right\}
$$

The jumps in temperature and stress found here agree with those derived in the more restrictive analysis of McCarthy and O'Leary [15].

The evolution of discontinuities in the field variables in a thermoviscoelastic medium is nearly the same as that in a thermoelastic medium, the one difference being that the decay factor $w$ is modified in a thermoviscoelastic material. It is evident from (3.32) that in a thermoviscoelastic medium the expression for $w$ contains two terms that are absent when the medium is thermoelastic. The term representing the contribution of the stress-strain relaxation function $\beta_{1}$ generates an increase in $w$ at both wave fronts, but the term involving the stress-temperature relaxation function $\beta_{2}$ produces an increase in $w$ only at the slower wave front. At the faster wave front the effect of $\beta_{2}$ is to make $w$ smaller. 
In all other respects, jumps in the field variables propagate identically through thermoviscoelastic media as they do through thermoelastic media. For example, the coupling between thermal and mechanical disturbances does not change in thermoviscoelastic materials. When $\alpha_{0}^{0}<1+\delta$ the fast wave is essentially mechanical in nature near $x=0$, whereas the slow wave is essentially a thermal wave. When $\alpha_{0}^{0}>1+\delta$ the situation is reversed. The fast wave is essentially thermal while the slow wave is mechanical. These conclusions follow from the argument used by Achenbach [6] in the simpler case of a thermoelastic material that conducts heat according to the Maxwell-Cattaneo relation. As McCarthy and O'Leary [15] have pointed out, it is possible for the waves to change character after travelling sufficiently far from the boundary $x=0$. For example, when $\alpha_{0}^{0}<1+\delta$ it is clear from the jump expressions (3.63)-(3.65) that, if $w_{2}>w_{1}$, there is a critical position beyond which the fast wave no longer carries the bulk of the jump in stress. On the other hand, if $w_{2}<w_{1}$, the slow wave ultimately ceases to carry the bulk of the jump in temperature. When $\alpha_{0}^{0}>1+\delta$ a similar situation arises. If $w_{2}<w_{1}$, the slower quasi-mechanical wave changes character, whereas the faster quasi-thermal wave is affected if $w_{2}>w_{1}$.

The techniques used in this paper not only yield information about the evolution of discontinuities in the field variables at the wave fronts but also provide a description of the behavior of the field variables behind the wave fronts. The solutions found here are ideally suited to numerical evaluation on account of their recursive properties. We present results for the case in which the relaxation functions $\beta_{1}(t), \beta_{2}(t), \beta_{3}(t)$, and $\alpha(t)$ are those discussed in the next section.

4. A sample problem. In this section we examine a problem in which the relaxation functions for the thermoviscoelastic half-space are given explicitly by

$$
\left.\begin{array}{l}
\beta_{1}(t)=m+(1-m) e^{-t / \tau_{1}}, \quad \beta_{2}(t)=1, \\
\beta_{3}(t)=1, \quad \alpha(t)=\frac{1}{\tau_{0}} e^{-t / \tau_{0}} .
\end{array}\right\}
$$

The relaxation functions defined in (4.1) are dimensionless. The function $\alpha(t)$ represents the Maxwell-Cattaneo model of heat conduction, with $\tau_{0}$ now the nondimensional thermal relaxation time. The function $\beta_{1}(t)$ models the viscoelastic response of the material as that of a standard linear solid: $m$ is the ratio of the relaxed elastic modulus to the instantaneous modulus and $\tau_{1}$ is the nondimensional viscoelastic relaxation time. In the limiting case $m \rightarrow 1, \tau_{1} \rightarrow 0$ the response of the material becomes elastic. Then the corresponding constitutive equations reduce to those of the linear theory of Lord and Shulman [4].

The Taylor series expansions about $t=0$ of the relaxation functions in (4.1) have the following coefficients:

$$
\left.\begin{array}{c}
\beta_{1.0}^{0}=\beta_{2,0}^{0}=\beta_{3.0}^{0}=1, \quad \alpha_{0}^{0}=\frac{1}{\tau_{0}}, \\
\beta_{2, i}^{0}=\beta_{3, i}^{0}=0, \quad \beta_{1, i}^{0}=(1-m)(-1)^{i} \tau_{1}^{-i}, \quad \alpha_{i}^{0}=(-1)^{i} \tau_{0}^{-(i+1)}, \quad i=1,2, \ldots .
\end{array}\right\}
$$


Therefore wave fronts travel through the thermoviscoelastic medium defined by these relaxation functions with the velocities $v_{1}=1 / S_{1}^{\prime}$ and $v_{2}=1 / S_{2}^{\prime}$, where

$$
\left.\begin{array}{l}
S_{1}^{\prime}=\left[\frac{\tau_{0}}{2}\left(1+\delta+\frac{1}{\tau_{0}}+\Gamma^{1 / 2}\right)\right]^{1 / 2}, \\
S_{2}^{\prime}=\left[\frac{\tau_{0}}{2}\left(1+\delta+\frac{1}{\tau_{0}}-\Gamma^{1 / 2}\right)\right]^{1 / 2},
\end{array}\right\}
$$

and

$$
\Gamma=\left(\frac{1}{\tau_{0}}+\delta-1\right)^{2}+4 \delta
$$

Jumps in the field variables at the wave fronts decay exponentially at a rate determined by the factors

$$
\left.\begin{array}{l}
w_{1}=\frac{\left(\left(S_{1}^{\prime}\right)^{2}-1\right)}{2 S_{1}^{\prime} \Gamma^{1 / 2}}\left[\frac{\left(S_{1}^{\prime}\right)^{2}}{\tau_{0}^{2}}+\frac{(1-m)}{\delta \tau_{1}}\left(1-\left(S_{1}^{\prime}\right)^{2} / \tau_{0}\right)^{2}\right], \\
w_{2}=\frac{\left(1-\left(S_{2}^{\prime}\right)^{2}\right)}{2 S_{2}^{\prime} \Gamma^{1 / 2}}\left[\frac{\left(S_{2}^{\prime}\right)^{2}}{\tau_{0}^{2}}+\frac{(1-m)}{\delta \tau_{1}}\left(1-\left(S_{2}^{\prime}\right)^{2} / \tau_{0}\right)^{2}\right] .
\end{array}\right\}
$$

The second term in each formula in (4.5) vanishes in the limiting case $m \rightarrow 1, \tau_{1} \rightarrow 0$, that is, in the elastic case.

The solutions to the two canonical boundary-value problems considered in the previous section remain those given by (3.60) and (3.61), but now with $S_{l}^{\prime}$ given by (4.3) and $w_{l}$ given by (4.5). The coefficients $t_{k j}$ and $u_{k j}$ are determined recursively from (3.46) and (3.47), with (3.57) providing $\bar{T}_{j}^{l}$ and $\bar{U}_{j}^{l}$, subject to (4.2). The starting values $\bar{T}_{0}^{l}$ and $\bar{U}_{1}^{l}$ are the same as those in the thermoelastic case [19], namely,

$$
\left.\begin{array}{l}
\bar{T}_{0}^{1}=\frac{1}{2}\left[1-\left(\frac{1}{\tau_{0}}+\delta-1\right) \Gamma^{-1 / 2}\right], \quad \bar{T}_{0}^{2}=\frac{1}{2}\left[1+\left(\frac{1}{\tau_{0}}+\delta-1\right) \Gamma^{-1 / 2}\right], \\
\bar{U}_{1}^{1}=-\frac{1}{S_{1}^{\prime} \Gamma^{1 / 2}}, \quad \bar{U}_{1}^{2}=\frac{1}{S_{2}^{\prime} \Gamma^{1 / 2}}, \quad \text { for problem } 1,
\end{array}\right\}
$$

and

$$
\left.\begin{array}{l}
\bar{T}_{0}^{1}=-\bar{T}_{0}^{2}=\delta \Gamma^{-1 / 2}, \quad \bar{U}_{1}^{1}=-\left[1+\left(\frac{1}{\tau_{0}}+\delta-1\right) \Gamma^{-1 / 2}\right] / 2 S_{1}^{\prime}, \\
\bar{U}_{1}^{2}=-\left[1-\left(\frac{1}{\tau_{0}}+\delta-1\right) \Gamma^{-1 / 2}\right] / 2 S_{2}^{\prime}, \quad \text { for problem 2. }
\end{array}\right\}
$$

The observation that, for the type of material considered in this section, the quantities $q_{1, k, j}$ and $q_{2, k, j}$ themselves satisfy recursion relations facilitates the efficient computation of the coefficients $t_{k j}$ and $u_{k j}$ from (3.46) and (3.47). These recursion relations are

$$
q_{1, k, j}=\left\{\begin{aligned}
-\frac{1}{\tau_{1}} q_{1, k, j-1}+\frac{1}{\tau_{1}^{2}}\left[\tau_{1}^{2} E_{w}^{2}+2 S^{\prime}(1-m) \tau_{1} E_{w^{\prime}}+\left(S^{\prime}\right)^{2}(1-m)\right] u_{k j} \\
\quad+\frac{m}{\tau_{1}} E_{w^{2}}^{2} u_{k, j-1}, \quad 0 \leq k \leq j-1, \\
\quad k<0 \text { or } k>j-1,
\end{aligned}\right.
$$


and

$$
q_{2, k, j}=\left\{\begin{array}{l}
-\frac{1}{\tau_{0}} q_{2, k, j-1}+\frac{1}{\tau_{0}^{3}}\left[\tau_{0}^{2} E_{w}^{2}+2 S^{\prime} \tau_{0} E_{w}+\left(S^{\prime}\right)^{2}\right] t_{k, j-1}, \quad 0 \leq k \leq j-1, \\
0, \quad k<0 \text { or } k>j-1 .
\end{array}\right.
$$

In order to illustrate our results graphically, we have numerically evaluated the formal wave front expansions that give the solution to problem 1 and plotted the products of the computations. The series are summed using the same technique that was employed in [19]. For each set of values of the parameters $\delta, \tau_{0}, \tau_{1}$, and $m$, the series solution is summed using 40 terms. For each case the output using 40 terms was in agreement with the output using fewer terms over a clearly defined range of $t$ for fixed $x$. This technique indicates clearly the length of time after the arrival of the wave front for which the series solutions are convergent. The influence of the mechanical and thermal parameters on the solutions at and behind the wave fronts is illustrated in Figures 1-5.
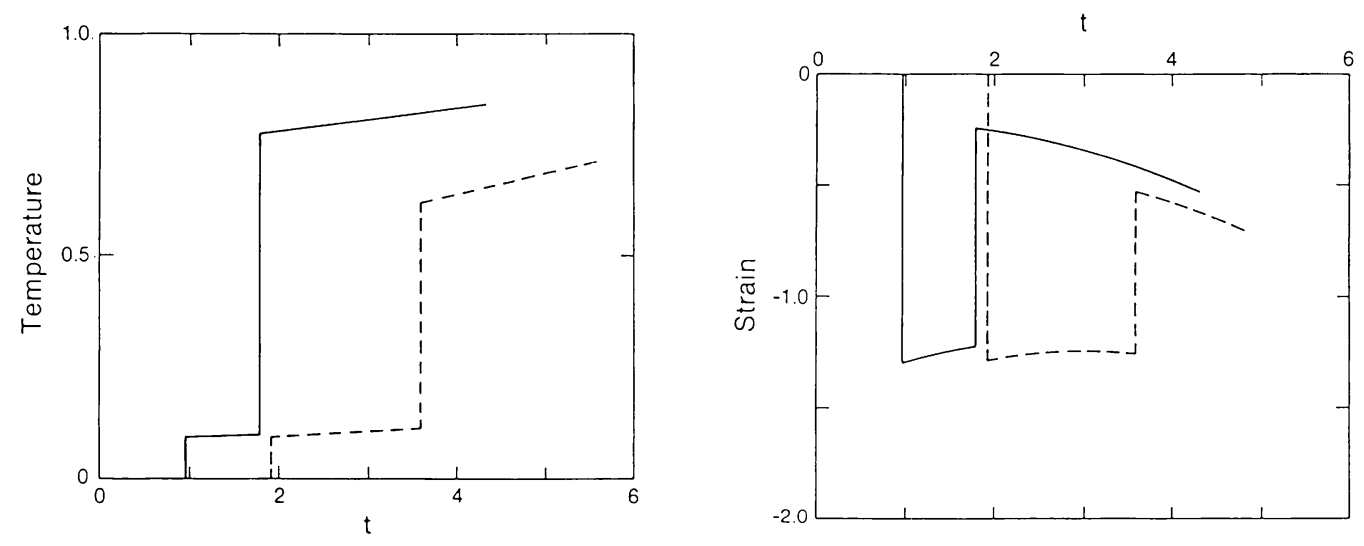

FIG. 1. Variation of (a) nondimensional temperature and (b) nondimensional strain with nondimensional time at the nondimensional stations $x=1.0(-)$, $2.0(--)$ for $\delta=0.05, \tau_{0}=3.0, \tau_{1}=5.0, m=0.95$.

In Fig. 1, the dimensionless temperature and strain are plotted for typical values of $\delta, \tau_{0}, \tau_{1}$, and $m$. The values of the thermoelastic coupling constant $\delta$ and the dimensionless relaxation time $\tau_{0}$ are chosen to be of the same order of magnitude as the values used in $[9,10,14,19]$. The values of the viscoelastic parameters $\tau_{1}$ and $m$ represent a material in which mechanical relaxation is not negligible. It is clear from Fig. 1 that the amplitude of the discontinuity at the slow wave front decays more rapidly with distance than the amplitude of the discontinuity at the fast wave front; that is, the quasi-thermal wave decays more rapidly with distance than the quasi-mechanical wave. The dependence of the temperature and strain on $\tau_{1}$ and $m$ is depicted in Figs. 2 and 3, respectively. Although changes in the viscoelastic parameters have no noticeable effect on the jumps at the wave fronts, some variation in the solutions with $\tau_{1}$ and $m$ can be observed behind the wave fronts. The consequences of varying $\delta$ and $\tau_{0}$ are demonstrated in Figs. 4 and 5, 

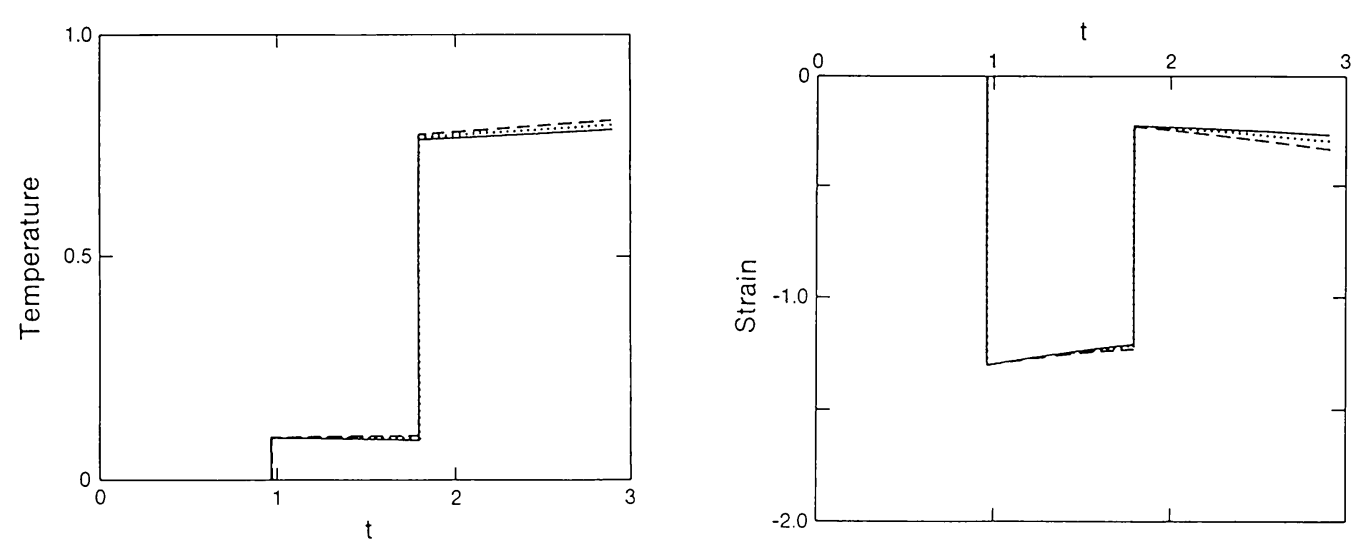

FIG. 2. Variation of (a) nondimensional temperature and (b) nondimensional strain with nondimensional time at the nondimensional station $x=1.0$ for $\delta=0.05$, $\tau_{0}=3.0, \tau_{1}=0, m=1.0(-)$, and $\tau_{1}=5.0(--), 10.0(\cdot), m=0.95$.
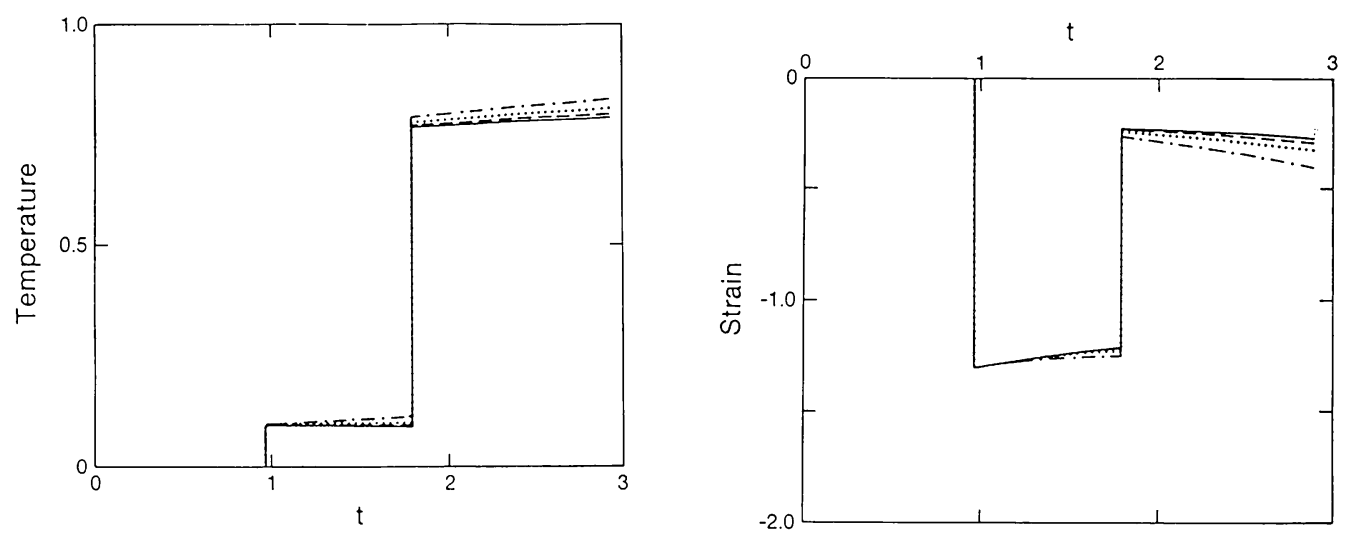

FIG. 3. Variation of (a) nondimensional temperature and (b) nondimensional strain with nondimensional time at the nondimensional station $x=1.0$ for $\delta=0.05$, $\tau_{0}=3.0, \tau_{1}=0, m=1.0(-)$, and $\tau_{1}=10.0, m=0.95(--), 0.9(\cdot), 0.8(-\cdot-)$.

respectively. These results are in agreement with those obtained in the thermoelastic case [19].

5. Discussion. In this paper we have presented methods for solving the integrodifferential equations of a linear theory governing the propagation of disturbances in a homogeneous, thermoviscoelastic medium. The methods are formal and directly yield complete asymptotic wave front expansions. The expansions are given explicitly via a set of recursion relations for the coefficients that enable one to determine an arbitrary number of terms in the solutions. The technique is suitable for numerical computations. The procedure is illustrated here for a simple but important example.

The general results obtained here regarding the propagation characteristics of transients in thermoviscoelastic materials confirm those reported previously by McCarthy and O'Leary [15]. The application of a mechanical or thermal disturbance in a onedimensional medium gives rise to two wave fronts in which mechanical and thermal 

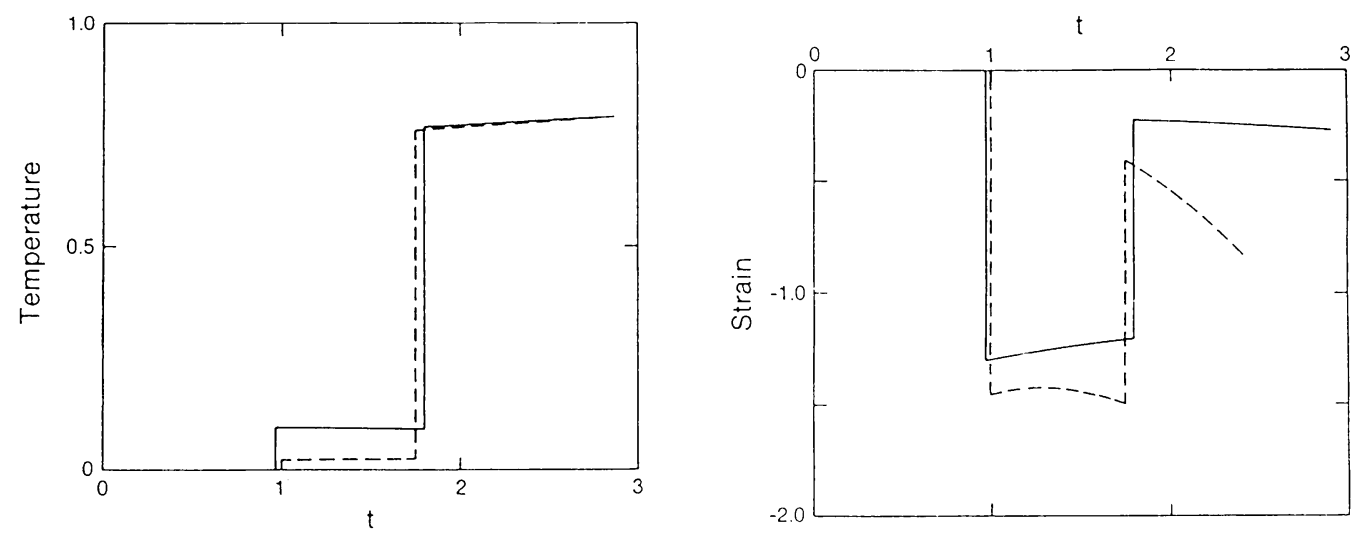

FIG. 4. Variation of (a) nondimensional temperature and (b) nondimensional strain with nondimensional time at the nondimensional station $x=1.0$ for $\delta=0.05(-)$, $0.01(--), \tau_{0}=3.0, \tau_{1}=10.0, m=0.99$.
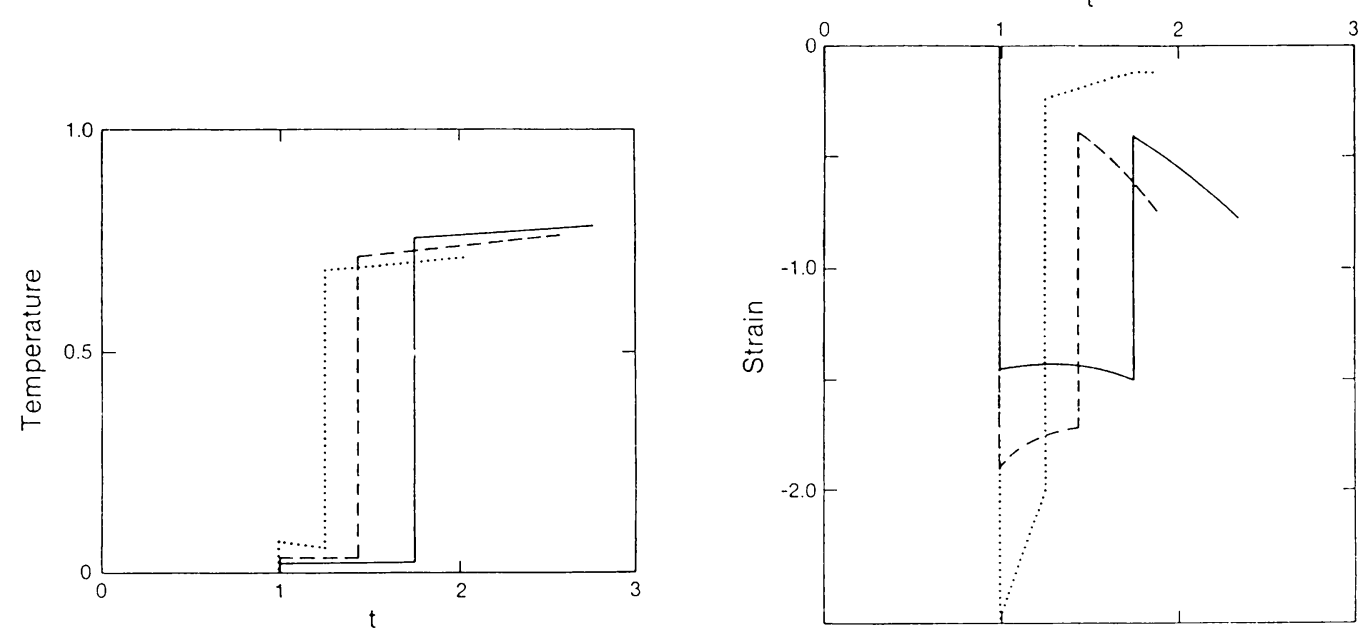

FIG. 5. Variation of (a) nondimensional temperature and (b) nondimensional strain with nondimensional time at the nondimensional station $x=1.0$ for $\delta=0.01$.

$$
\tau_{0}=3.0(-), 2.0(--), 1.5(. \cdot), \tau_{1}=10.0, m=0.99 \text {. }
$$

effects are coupled. One wave front travels with a velocity greater than the instantaneous isothermal velocity of longitudinal elastic waves and the other with a velocity smaller than the elastic speed. When $\alpha_{0}^{0}<1+\delta$, where $\delta$ is a thermoelastic coupling constant and $\alpha_{0}^{0}$ the instantaneous value of the heat flux relaxation function, the fast wave is quasi-mechanical and the slow wave quasi-thermal. For $\alpha_{0}^{0}>1+\delta$ the situation is reversed. The discontinuities in the field variables at the wave fronts decay exponentially with distance from the source of the disturbance. The rate of decay is affected strongly by the mechanical and thermal relaxation characteristics of the medium.

Unlike the techniques used by McCarthy and O'Leary, however, the methods presented here are not limited in the range of their validity to the sites of the wave 
fronts. They are capable, as well, of describing the behavior of the field variables for some distance behind the wave fronts.

\section{REFERENCES}

[1] C. Cattaneo, Sur une forme de l'équation de la chaleur éliminant le paradox d'une propagation instantanée, C. R. Acad. Sci. Paris 247, 431-433 (1958)

[2] M. E. Gurtin and A. C. Pipkin, A general theory of heat conduction with finite wave speeds, Arch. Rat. Mech. Anal. 31, 113-126 (1968)

[3] V. K. Agarwal, Some remarks on a generalized heat conduction equation, Amer. J. Phys. 49, 503-504 (1981)

[4] H. W. Lord and Y. Shulman, A generalized dynamical theory of thermoelasticity, J. Mech. Phys. Solids 15, 299-309 (1967)

[5] P. J. Chen and M. E. Gurtin, On second sound in materials with memory, Z. Angew. Math. Phys. 21, 232-241 (1970)

[6] J. D. Achenbach, The influence of heat conduction on propagating stress jumps, J. Mech. Phys. Solids 16, 273-282 (1968)

[7] F. R. Norwood and W. E. Warren, Wave propagation in the generalized dynamical theory of thermoelasticity, Quart. J. Mech. Appl. Math. 22, 283-290 (1969)

[8] E. B. Popov, Dynamic coupled problem of thermoelasticity for a half-space taking account of the finiteness of the heat propagation velocity, J. Appl. Math. Mech. (PMM) 31, 349-356 (1967)

[9] H. W. Lord and A. A. Lopez, Wave propagation in thermoelastic solids at very low temperature, Acta Mech. 10, 85-98 (1970)

[10] Y. H. Pao and D. K. Banerjee, Thermal pulses in dielectric crystals, Lett. Appl. Eng. Sci. 1, 33-41 (1973)

[11] D. K. Banerjee and Y. H. Pao, Thermoelastic waves in anisotropic solids, J. Acoust. Soc. Amer. 56, 1444-1454 (1974)

[12] A. Nayfeh and S. Nemat-Nasser, Thermoelastic waves in solids with thermal relaxation, Acta Mech. 12, 53-69 (1971)

[13] A. H. Nayfeh, Transient thermo-elastic waves in a half-space with thermal relaxation, Z. Angew. Math. Phys. 23, 50-68 (1972)

[14] A. H. Nayfeh, Propagation of thermoelastic disturbances in non-Fourier solids, AIAA J. 15, 957-960 (1977)

[15] M. F. McCarthy and P. M. O'Leary, Wave propagation in linear thermoviscoelastic materials, J. Thermal Stresses 5, 347-364 (1982)

[16] M. E. Gurtin, Time-reversal and symmetry in the thermodynamics of materials with memory, Arch. Rat. Mech. Anal. 44, 387-399 (1972)

[17] F. Mainardi, On thermal waves in generalized linear theory of heat conduction, CISM Symposium on thermomechanics in solids, Udine, Italy (1974)

[18] T. B. Moodie and R. J. Tait, On thermal transients with finite wave speeds, Acta Mech. 50, 97-109 (1983)

[19] R. P. Sawatzky and T. B. Moodie, On thermoelastic transients in a general theory of heat conduction with finite wave speeds, Acta Mech. 56, 165-187 (1985)

[20] R. M. Christensen, Theory of viscoelasticity: an introduction, 2nd ed., Academic Press, New York, 1982

[21] P. J. Chen and J. W. Nunziato, Thermodynamic restrictions on the relaxation functions of the theory of heat conduction with finite wave speeds, Z. Angew. Math. Phys. 25, 791-797 (1974)

[22] R. Courant and D. Hilbert, Methods of mathematical physics, Vol. II, Interscience, New York, 1962 